Editorial

\title{
Research on Efficient Data Forwarding in Vehicular Networks
}

\author{
Syed Hassan Ahmed $\mathbb{D}^{1}$, Ali K. Bashir $\mathbb{D}^{2}{ }^{2}$ Mohamed Elhoseny, ${ }^{3}$ Wael Guibene, ${ }^{4}$ \\ and Safdar Hussain Bouk ${ }^{5}$ \\ ${ }^{1}$ Georgia Southern University, Statesboro, GA 30460, USA \\ ${ }^{2}$ Manchester Metropolitan University, Manchester M15GD, UK \\ ${ }^{3}$ Mansoura University, El Gomhouria St, Mansoura, Dakahlia 35516, Egypt \\ ${ }^{4}$ Amazon Lab126, Sunnyvale, CA 94089, USA \\ ${ }^{5}$ DGIST, Daegu 41566, Republic of Korea \\ Correspondence should be addressed to Syed Hassan Ahmed; sh.ahmed@ieee.org
}

Received 16 April 2019; Accepted 17 April 2019; Published 2 May 2019

Copyright (C) 2019 Syed Hassan Ahmed et al. This is an open access article distributed under the Creative Commons Attribution License, which permits unrestricted use, distribution, and reproduction in any medium, provided the original work is properly cited.

Recent advances in vehicular communications and intelligent transportation systems (ITS) intend to trim down the fuel expenditure by avoiding congested traffic, enhancement of traffic safety, and initiating new application, that is, mobile infotainment [1]. Commonly, we have three types of vehicle communication models, that is, vehicle-to-vehicle $(\mathrm{V} 2 \mathrm{~V})$, vehicle-to-infrastructure (V2I), and vehicle-to-roadside (V2Rs) communications [2]. Due to the rapid growth in this field, many research constraints need to be addressed, for example, reliability and latency, appropriate scalable design of MAC and routing protocols, performance and adaptability to the changes in environment (node density and oscillation in network topology), and evaluation and validation of vehicular communication protocols under the umbrella of coherent assumptions using simulation methodologies.

This special issue aimed to emphasize the latest achievements to identify the robust and efficient data forwarding techniques in vehicular communications and similar networks. We believe that the high-quality accepted papers in this special issue will add new insight to the readers' knowledge.

The Internet of Vehicles (IoV) is a substrate for safe, efficient, and green ITS. Due to highly dynamic nature of the network, the reliable and timely dissemination of the safety and warning information, in basic safety messages (BSM), is a challenging task [3]. In the first paper entitled " $\mathrm{A}$ Local Information Sensing-Based Broadcast Scheme for Disseminating Emergency Safety Messages in IoV," the authors proposed the Local Topology Information Sensing technology-based broadcast (LISCast) protocol to address the slow response and local broadcast storm problem by employing the probability-based forwarding scheme. The results show that the proposed LISCast achieves low delay and alleviates broadcast redundancy in dynamic topology vehicular network. Furthermore, the authors in "A Heterogeneous IoV Architecture for Data Forwarding in Vehicle to Infrastructure Communication" proposed the architecture that ensures the reliable data transmission in IoV. As modern vehicles are equipped with multiple wireless interfaces, e.g., Wireless Access in Vehicular Environment (WAVE), Long-Term Evolution (LTE), Long-Range Wireless Fidelity (Wi-Fi), and so forth, the proposed architecture provides mechanism to select best wireless interface among the available ones to ensure data communication reliability and seamless connectivity, avoiding single point of failure. The next paper entitled "A Novel Method for Predicting Vehicle State in Internet of Vehicles" in the IoV domain of this special issue proposed the vehicle state prediction scheme. The authors used a decision tree method to recognize the driving behaviour and vehicle sate on different road segments.

In addition to the IoV related papers [4], there are some contributions by the authors that address communication problems in the specific vehicular communication scenarios, e.g., V2V, V2I, and V2X. For example, in the paper entitled "Integrated Packet Classification to Support Multiple Security Policies for Robust and Low Delay V2X Services," the authors proposed the memory efficient packet classification 
algorithm for V2X scenario. The packet classification is necessary to avoid sophisticated cyber attacks that impact on the data delivery delay constraint. The authors in "Predicting the Route the Longest Lifetime and the Data Packet Delivery Time between Two Vehicles in VANET" proposed two schemes that predict packet delivery time by utilizing the vehicle density and vehicles' mobility information to determine the route lifetime for highway traffic scenarios. The performance and quality of data communication in vehicular network deteriorates due to intrinsic channel characteristics such as multipath fading and shadowing. Therefore, the authors in "Rate Adaptation Mechanism with Available Data Rate Trimming and Data Rate Information Provision for V2I Communications" proposed two data rate adaptation schemes, available data rate trimming and data rate information provision schemes, to increase communication performance of the network. The authors in the paper entitled "CMD: A Multichannel Coordination Scheme for Emergency Message Dissemination in IEEE 1609.4" proposed the safety message information disseminate scheme over multiple IEEE 1609.4 channels, control channel (CCH), and the service channels (SCHs).

In addition to the conventional networking technologies, authors have also investigated the future Internet architectures in vehicular networks. The authors in "Security \& Privacy Issues in Vehicular Named Data Networks: An Overview" overviewed the security and privacy issues in Named-Data Networking (NDN) enabled vehicular networks. The NDN-based vehicular networks are at their earlier stage and require more detailed investigation. To this regard, authors in the work entitled "Cluster-Based Device Mobility Management in Named Data Networking for Vehicular Networks," proposed the cluster-based device mobility management (CB-DMM) system for NDN-based vehicular networks. Each cluster is managed by the cluster head, which maintains the route information for its cluster members. The content information is shared by the content producer, and this content location information is mapped and managed by the clusters. The content requesting Interest messages are forwarded based on that mapping information in the network. The results showed that the proposed CBDMM has high content request satisfaction ratio.

Furthermore, in the paper entitled "Smartwatch-Based Legitimate User Identification for Cloud-Based Secure Services," the authors have proposed the smart watch-based activity recognition and gait-based legitimate user identification based on the time and frequency domain sensory information of user activity. In the next work, "A New Distance Vector-Hop Localization Algorithm Based on HalfMeasure Weighted Centroid," a new distance vector-hop localization algorithm using the half-measure weighted centroid for wireless sensor networks. Using two-dimensional position distribution, the algorithm first constructs the approximate communication radius and network connectivity. The algorithm then corrects the distance between the beacon node and its neighbours to increase the jump distance accuracy to compute the optimized shortest path.

In the last paper of this special issue, the authors have proposed a Bloch chain-based incentive mechanism for data storing wireless sensor nodes in the paper entitled "Incentive Mechanism of Data Storage Based on Blockchain for Wireless Sensor Networks." More data-storing nodes receive more incentive because they offer their limited storage resource for other nodes in the network. The prime objective of the proposed scheme is to conserve the storage space of the wireless sensor nodes.

\section{Conflicts of Interest}

The guest editors declare that they have no conflicts of interest.

\section{Acknowledgments}

The guest editors would like to sincerely thank all the authors who submitted their contributions in our special issue. They would also like to thank all our reviewers and sincerely acknowledge their contribution that has been substrate to the success of this special issue.

Syed Hassan Ahmed Ali K. Bashir

Mohamed Elhoseny Wael Guibene

Safdar Hussain Bouk

\section{References}

[1] C. A. Kerrache, N. Lagraa, R. Hussain et al., "TACASHI: trustaware communication architecture for social internet of vehicles," IEEE Internet of Things Journal, p. 1, 2018.

[2] S. H. Bouk, S. H. Ahmed, D. Kim, K.-J. Park, Y. Eun, and J. Lloret, "LAPEL: hop limit based adaptive PIT entry lifetime for vehicular named data networks," IEEE Transactions on Vehicular Technology, vol. 67, no. 7, pp. 5546-5557, 2018.

[3] M. F. Majeed, S. H. Ahmed, and M. N. Dailey, "Enabling pushbased critical data forwarding in vehicular named data networks," IEEE Communications Letters, vol. 21, no. 4, pp. 873-876, April 2017.

[4] J. Chen, G. Mao, C. Li, and D. Zhang, "A topological approach to secure message dissemination in vehicular networks," IEEE Transactions on Intelligent Transportation Systems, pp. 1-14, 2019. 


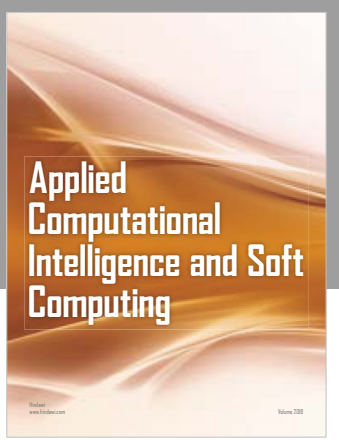

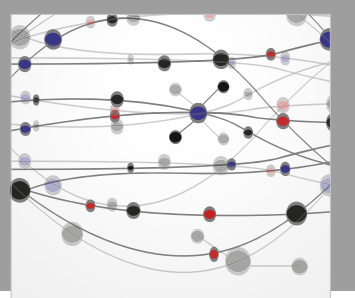

The Scientific World Journal
Submit your manuscripts at

Computing
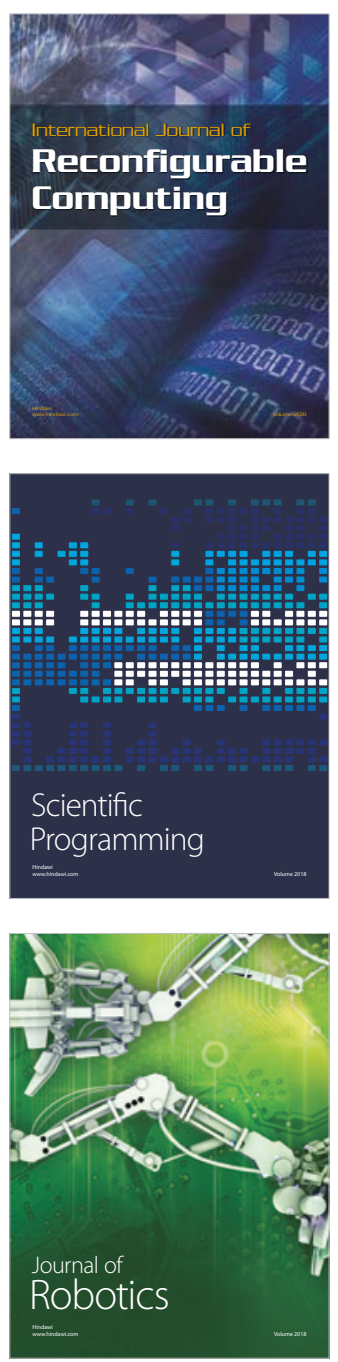

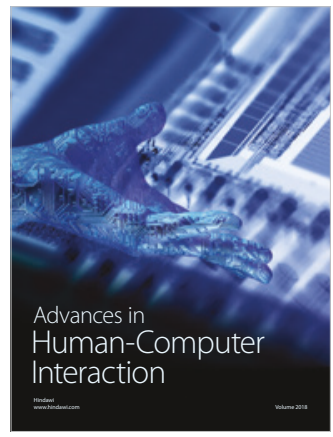

Human-Compute

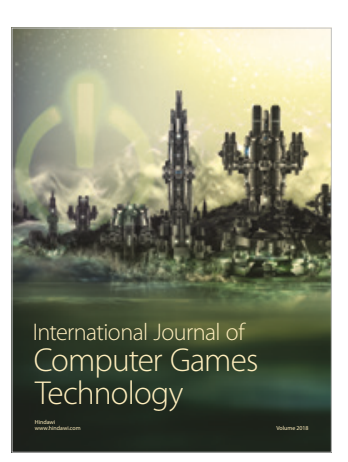

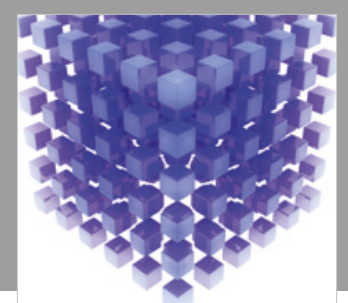

Mathematical Problems in Engineering

\section{Engincering}
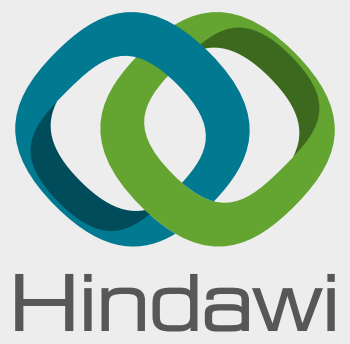

www.hindawi.com
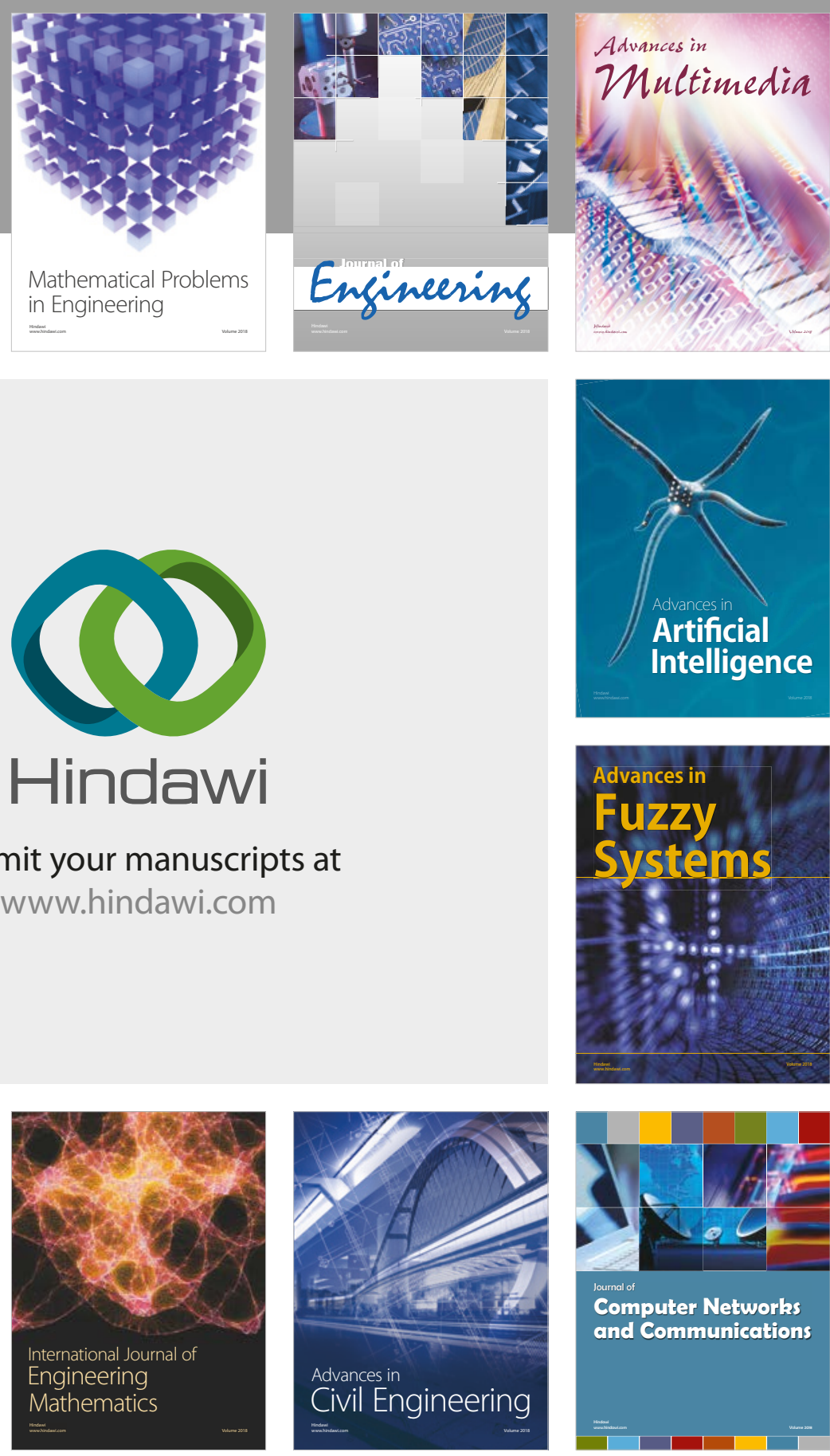

Computer Networks and Communications

Multimedia
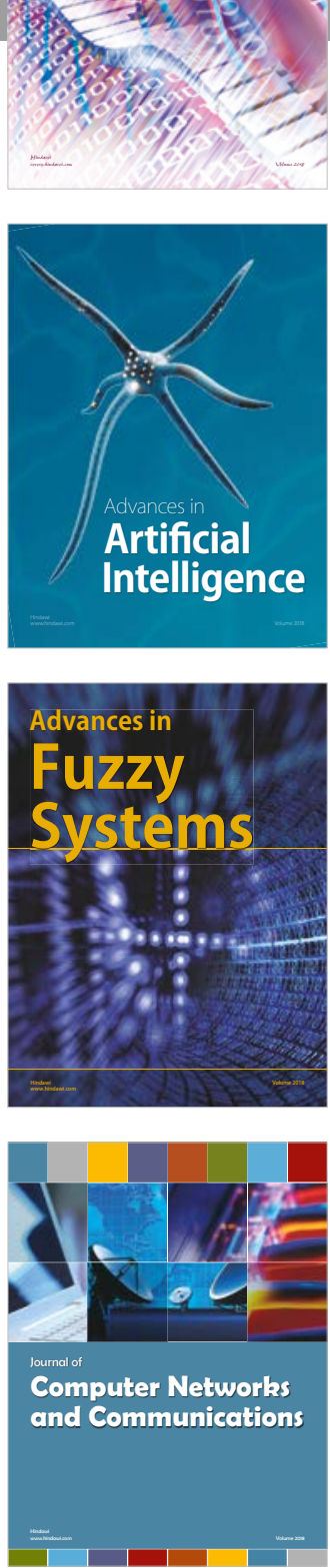

Advances in

Modelling \&

Simulation

in Engineering

interaction

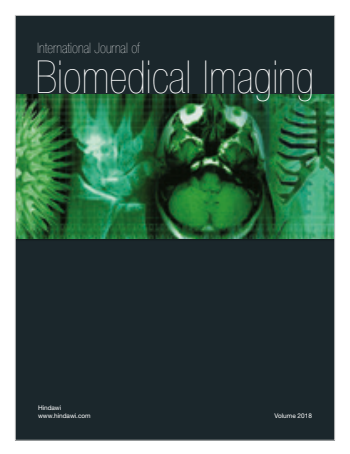

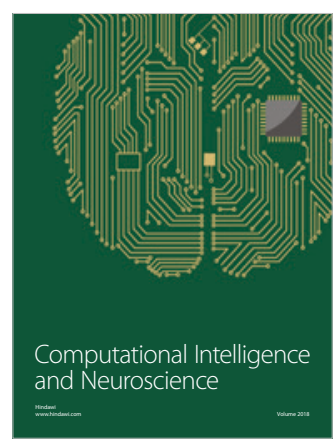

